\title{
Low Temperature Photoluminescence in Ultra-Thin Germanium Quantum Wells
}

\author{
P. A. M. Rodrigues, \\ Instituto de Física, Universidade de Brasilia, Cx.Postal 04455, CEP 70910-900, Brasília, DF, Brazil \\ M. A. Araújo-Silva, \\ Departamento de Física, Universidade Federal do Ceará, Fortaleza CE, Brazil \\ G. A. Narvaez, F. Cerdeira, \\ Instituto de Física, Universidade Estadual de Campinas, Campinas SP, Brazil \\ and J. C. Bean \\ Department of Electrical Engineering, \\ University of Virginia. Charlottesville VA 22903-2442, USA
}

Received 01 June, 1999

\begin{abstract}
We measured the photoluminescence (PL) spectra of a series of Ge $e_{n}$ quantum wells as a function of temperature, from $2 \mathrm{~K}$ to $50 \mathrm{~K}$. The PL spectra at $2.1 \mathrm{~K}$ are dominated by broad emission lines, which can be interpreted as recombination across the indirect gap of the Si/Ge microstructure and are strongly influenced by the interface morphology of each sample. Beyond $T \gtrsim 15 \mathrm{~K}$, all samples show identical spectra in which the broad structures are replaced by thin, strong lines. We interpret these changes as a quenching of the recpmbination across the gap PL of the microstructure and the appearance of defect-related peaks from the Si substrate.
\end{abstract}

\section{Introduction}

During the last decade $\Gamma$ there has been an increasing number of reports on the photoluminescence (PL) spectra of a variety of $\mathrm{Si} / \mathrm{Ge}$ microstructures [1-10]. The low temperature PL spectra ( $T \lesssim 4 \mathrm{~K}$ ) of a wide variety of $\mathrm{Si} / \mathrm{Ge}$ microstructuresTranging from $\mathrm{Si}_{m} \mathrm{Ge}_{n}$ superlattices [2] $\Gamma \mathrm{Si} / \mathrm{Ge}_{n}$ quantum wells [3-5] to $\mathrm{Ge}_{x} \mathrm{Si}_{1-x} / \mathrm{Si}$ quantum wells $\Gamma$ superlattices and quantum dots [617] $\Gamma$ are remarkably similar. They consist of two relatively broad peaks (across the gap recombination and phonon replica $\Gamma$ respectively) $\Gamma$ or a single very broad band at lower photon energy $\Gamma$ which appears to be related to interface defects created by Ge segregation [6]. All $\mathrm{Si} / \mathrm{Ge}$ microstructures grown by molecular beam epitaxy (MBE) seem to have a common feature: a thin alloy layer (1-2 monolayers) produced by interdiffusion during growth [15Il1]. This layer could influence the PL spectra of samples with different architectures $\Gamma$ giving them a characteristic signature Teither by disorder- induced exciton localization or by providing the conduction band minimum for the microstructure. As the temperature increases $\Gamma$ the reported PL spectra show two different characteristics. While some maintain their general lineshape at higher temperatures [374 I8] Tothers exhibit qualitatively different shapes [9 $110 \pi 2]$. Again the same behavior is exhibited by samples of different architectures within each type of behavior.

We measured the photoluminescence (PL) spectra of a series of $\mathrm{Ge}_{n}$ multiple quantum wells (MQWs) in the temperature range between $2 \mathrm{~K}$ and $50 \mathrm{~K}$. The $\mathrm{PL}$ spectra at $2.1 \mathrm{~K}$ are dominated by broad emission lines in the photon energy range between 0.80 and $0.97 \mathrm{eV}$. These lines can be interpreted as recombination across the indirect gap of the $\mathrm{Si} / \mathrm{Ge}$ microstructure and are strongly influenced by the interface morphology of each sample [5]. Beyond $T \sim 15 \mathrm{~K}$ Tall samples show identical spectra in which the broad structures are quenched $\Gamma$ and thin strong lines dominate the spectra [12]. By comparing the PL spectra of the front (film) and back 
(substrate) end of one of our samplesTwe conclude that these thin lines originate in recombination at deep impurity complexes in the Si substrates and are unrelated to the microstructures deposited on them. We believe that this is also the explanation for similar high temperature PL lines reported by other authors in samples with different architecture [9П10].

\section{Experimental Details}

Our samples were grown by molecular beam epitaxy (MBE) at $\mathrm{T}=500^{\circ} \mathrm{C}$ on $\mathrm{Si}(001)$ substrates. They consist of a thick ( $800 \mathrm{~nm}) \mathrm{Si}$ buffer layer followed by 25 repetitions of $\mathrm{Ge}_{n}$ layers $(n=3 \Gamma 4 \Gamma 5$ and 6 monolayers $\Gamma \mathrm{ML}) \mathrm{T}$ separated from one another by $70 \mathrm{MLs}$ thick Si spacer-layers. Photoluminescence (PL) measurements were performed with the sample immersed in superfluid liquid He $(2.1 \mathrm{~K})$ or gas $(T \gtrsim 5 \mathrm{~K})$ Tusing approximately $30 \mathrm{Watts} / \mathrm{cm}^{2}$ of the $488 \mathrm{~nm}$ line of an Argon-ion laser as exciting radiation. Emitted light was filtered by a $0.5 \mathrm{~m}$ SPEX monochromator and detected with a cooled Ge detector.

\section{Results and Discussion}

Figure 1(a) displays low-temperature (2.1K) PL spectra of our $\mathrm{Si} / \mathrm{Ge}_{5} / \mathrm{Si} \mathrm{MQWTin}$ the photon energy range below $1 \mathrm{eV}$. This spectrum shows a broadband emission (BB-PL) centered at $\hbar \omega \sim 0.8 \mathrm{eV}$ and a narrower-band doublet (NB-PL) at slightly higher photon- energy. The $\mathrm{BB}-\mathrm{PL}$ is more or less identical in position and intensity in all MQW samplesT while the NB-PL exhibits confinement shifts going from $n=5$ to $n=6$ [5]. Similar bands appear in the spectra of $\mathrm{Si} / \mathrm{Ge}_{x} \mathrm{Si}_{1-x} \mathrm{QWs}$ and SLsT where BB or NB PL-lines are observed depending on the density of defects present in the alloy layer [6]. The NB-PL doublet is produced by no-phonon recombination across the indirect gap of the alloy and its phonon replicaT respectively [6]. The resemblance between the PL spectra of alloy and pure Ge QWs becomes more marked when we notice that the energy separation between the no-phonon emission line and its phonon-assisted replica in the NB-PL of all these samples is $49 \mathrm{meV}[3-5]$ The frequency of the Si-Ge vibration of the alloy. The $\mathrm{Ge}_{n}$ QWs also contain alloy layersTwhether intentionally grown [4] or appearing spontaneously during growth at the $\mathrm{Si} / \mathrm{Ge}$ interface [35]. In the first case $T$ the conduction band minimum of the microstructure is located in the alloy layers sandwiching the Ge QWs [4]. In the second case [35] T this interfacial alloy layer probably mediates the recombination by disorder- induced exciton localization [11]. In both cases the valence band maximum is located in the Ge QWs [4]. HenceT the PL lines would exhibit the signature of alloy PL (general shape and peak separation)I while photon energy positions shift as quantum well width varies due to the different confinement energies of the holes in the Ge layers.

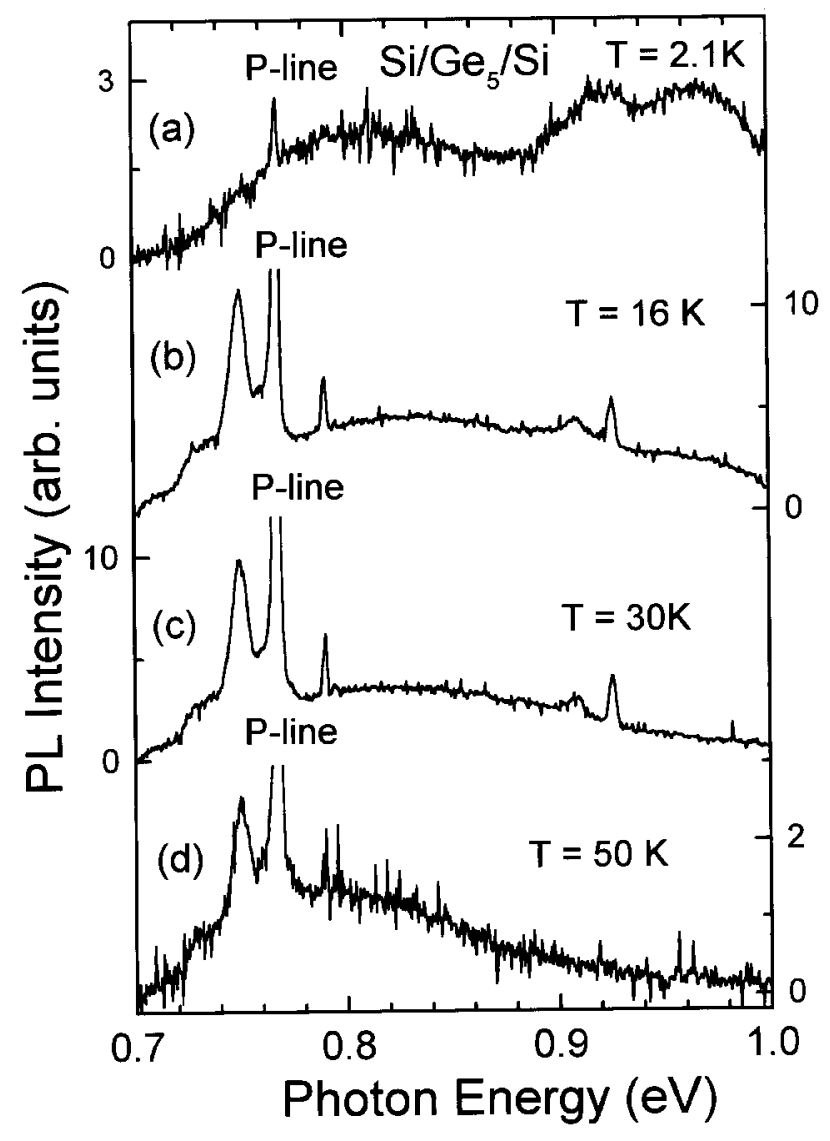

Figure 1. Photoluminescence (PL) spectra of the $\mathrm{Si} / \mathrm{Ge}_{5} / \mathrm{Si}$ sample at different temperatures.

At higher temperatures two types of behaviors are reported. Some [374I8] show spectra which resemble closely those taken at $T \lesssim 4 \mathrm{~K} \Gamma$ while others report spectra which suffer qualitative changes as the temperature increases [9П10]. Our samples exhibit the second type of behavior $\Gamma$ as exemplified in Figs 1(b) through (d) Twhere spectra for our sample with $n=5$ are displayed at different temperatures. Beyond $T \sim 15 \mathrm{~K}$ all our $\mathrm{Si} / \mathrm{Ge}_{n} / \mathrm{Si}$ samples show identical spectra $\mathrm{T}$ in which the broad structures are replaced by thinTstrong lines. A single sharp line (labeled P-line in Fig. 1) lo- 
cated at $0.767 \mathrm{eV}$ with a full width at half maximum of less than 2.3 meVTdominates the spectra [13]. Similar spectra have been reported in self-assembled Ge quantum dots [9] and in a rather large variety of $\mathrm{Si} / \mathrm{Ge}$ microstructuresTgrown by MBE on Si substrates $\Gamma$ with very different architecture [10]. The spectral lines are usually interpreted as recombination across the gap of the microstructure and conclusions about the sample structure and morphology (size uniformity of the quantum dots $\Gamma$ width of the quantum wellsTetc.) are drawn from the photon energy position and widths of the PL lines [9]. However Tthe ubiquity of these PL lines led De Padova et al. to suggest that they may not originate in the microstructures themselves $\mathrm{b}$ but rather are the product of recombination in some feature common to all these samples[such as the Si/Ge interface [10]. In this case $\Gamma$ the difference between the results just discussed and those of Gail et al [4] would reside in the interface quality of the samples. In the latterTthe PL of the microstructure persists even at high temperatures $\Gamma$ while in the other cases this PL is rapidly quenched and the spectrum is dominated by defect related luminescence. In an effort to clarify this issueTwe polished and etched the back (substrate) end of one of our samples $(n=5)$ and proceeded to study the PL spectra of both front and back ends of the same sample as a function of temperature [12]. We found that $\Gamma$ for $T \gtrsim 15 \mathrm{~K} \Gamma$ the spectra from the Si substrate are identical to those of the front end of all our samples. This is illustrated in Fig. $2 \Gamma$ where the spectra (at $16 \mathrm{~K}$ ) of the back (substrate) end of the sample $n=5$ and those of the front (film) end of our Si/Ge $/ \mathrm{Gi}_{n} / \mathrm{Si}$ samples are displayed. The fact that these thin, strong lines appear in exactly the same manner in all spectra unequivocally identifies them as the product of recombination taking place at the samples substrates, i.e., they are bulk Si lines. The inset in Fig. 2 shows the PL spectra at $T=2.1 \mathrm{~K}$ of the substrate (back end) and the film (front end) of the sample $n=5$. Here both spectra are quite different. Although the P-line $(\hbar \omega=0.767 \mathrm{eV})$ is already present in the spectrum of the QWT the NB- and BB-PL dominates this spectrum. In Table I we list the photon energy position $(\hbar \omega) \Gamma$ full width at half maximum $(\Gamma)$ Tand relative intensities (arbitrarily attributing an intensity of 100 units to the P-line at $0.767 \mathrm{eV}$ ) of the most prominent lines in the PL spectra at $T=16 \mathrm{~K}$ shown on Fig. 2 [13]. The various lines arising from recombination centers in bulk $\mathrm{Si}$ have been reported by several authors [14] and collected in the review of Davies [15]. The last column in Table I list the assignment given to PL lines of bulk $\mathrm{Si}$ appearing at the same photon energies (and with similar linewidth and relative intensities) as those in Fig. 2 [13]. This confirms our conclusion that the observed PL lines in the higher temperature spectra of our samples $\Gamma$ and probably also those of several other authors [9п0] Toriginate in recombination taking place at deep impurity centers in the Si substrate and are unrelated to the microstructure deposited on them.

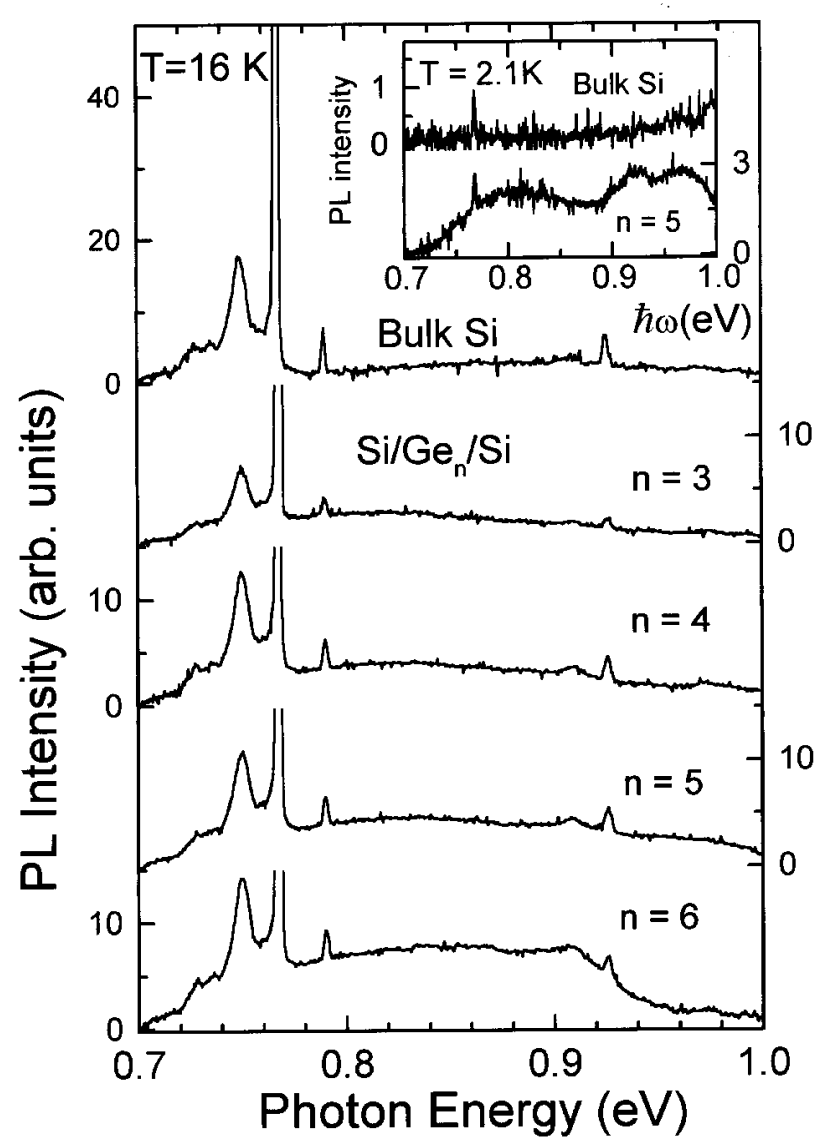

Figure 2. Photoluminescence (PL) spectra of the back (substrate) end of the sample with $n=5$ (top) and those of the front ends of all the $\mathrm{Si} / \mathrm{Ge}_{n} / \mathrm{Si} \mathrm{MQWs}$ at $16 \mathrm{~K}$. Inset shows the spectra of the back (top) and front ends of the $n=5$ sample at $2.1 \mathrm{~K}$.

In summary we have measured the PL spectra of a series of $\mathrm{Si} / \mathrm{Ge}_{n} / \mathrm{Si}$ samples $(n=3 \Gamma 4 \Gamma 5 \Gamma$ and 6$)$ in the temperature range $2 K<T<50 \mathrm{~K}$. The spectra for $T \gtrsim 15 \mathrm{~K}$ are dominated by thin $\Gamma$ strong lines $\Gamma$ which have been interpreted by some other authors as recombination across the bandgap of the microstructure. By comparing the PL spectra of the back (substrate) end of one sample with those taken from the front (film) end $\Gamma$ we identify these lines as recombination at deep centers in the Si substrate. 
Table I. Photon energies $(\hbar \omega) \Gamma$ linewidths $(\Gamma)$ and relative intensities (with that of the line at $0.767 \mathrm{eV}$ taken arbitrarily at 100) of the PL lines appearing in the $T=16 \mathrm{~K}$ spectra of the front and back ends of the $\mathrm{Si} / \mathrm{Ge}_{5} / \mathrm{Si}$ sample. The relative intensities have been corrected by the response of the experimental set up [13]. The last column gives the assignment of similar lines appearing in the PL spectra of bulk Si as listed in ref. [15].

\begin{tabular}{|c|c|c|}
\hline $\begin{array}{c}\hbar \omega(\mathrm{eV}) \\
{[\text { ГГनWHMTin meV] }}\end{array}$ & Relative Intensity & Assignment \\
\hline $\begin{array}{c}0.728 \\
{[\Gamma \sim 10]}\end{array}$ & 4.8 & LA sideband of P-line \\
\hline $\begin{array}{c}0.749 \\
{[\Gamma=8.3]}\end{array}$ & 18.1 & TA sideband of P-line \\
\hline $\begin{array}{c}0.767 \\
{[\Gamma \leq 2.3]}\end{array}$ & 100 & $\begin{array}{l}\text { P-line. No-phonon line of an Oxygen } \\
\text { defect or Carbon/Oxygen complex }\end{array}$ \\
\hline $\begin{array}{c}0.790 \\
{[\Gamma \leq 2.3]}\end{array}$ & 2.7 & $\begin{array}{l}\text { C-line. No-phonon line of a } \\
\text { Carbon/Oxygen complex }\end{array}$ \\
\hline $\begin{array}{c}0.908 \\
{[\Gamma=7.5]}\end{array}$ & 0.4 & TA sideband of H-line \\
\hline $\begin{array}{c}0.926 \\
{[\Gamma \leq 4.0]}\end{array}$ & 1.3 & $\begin{array}{l}\text { H-line. No-phonon line of a center } \\
\text { related to Carbon or Oxygen. }\end{array}$ \\
\hline
\end{tabular}

\section{Acknowledgments}

We acknowledge financial help $\Gamma$ in the form of research grants and fellowshipsTfrom FAPESPT FAPDF and $\mathrm{CNPq}$.

\section{References}

[1] The subject has been recently reviewed by F. Cerdeira in chapter 5 of Germanium silicon: Physics and Materials, edited by R. Hull and J.C. Bean, Semiconductors and Semimetals, Series editors R. K. Willardson and E. R. Weber Academic Press, New York, 1999) vol.56, p. $277-288$.

[2] U. Meczingar, G. Abstreiter, G. Olajos, J. Grimmeis, H. Kibbel, H. Prestig and E. Kasper, Phys. Rev. B 47, 4099 (1993), and references therein.

[3] J. Engvall, J. Olajos, H.Grimeiss, H. Kibbel and H. Prestig, Phys. Rev. B 51, 2001(1995).

[4] M. Gail, G. Abstreiter, J. Olajos, J. Engvall, H. Grimeiss, H.Kibbel and H. Prestig, Appl. Phys. Lett. 66, 2978 (1995).

[5] G. Narvaez, M. A. Araújo-Silva, F. Cerdeira and J. C.Bean, Solid State Commun. 107, 359 (1998).

[6] J. C. Sturm, A. St. Amour, Y. Lacroix and M. L. W. Thewalt, Appl. Phys. Lett. 64, 2291 (1994), and references therein.
[7] Y. S.Tang, S. Cai, G. Jin, J. Duan, K. L. Wang, H. M. Soyez and B.S. Dunn, Appl. Phys. Lett. 71, 2448 (1997).

[8] H. Sunamura, N.H.Usami and Y. Shiraki, Appl. Phys. Lett. 66, 3024 (1995).

[9] Xun Wang, Zui-min Jiang, Hai-jun Zhu, Fang Lu, Daming Huang, Xiaohan Liu, Chang-wu Hu, Yifan Chen and Takafumi Yao, Appl. Phys. Lett. 71, 3543 (1997).

[10] P. De Padova, P. Perfetti, R. Pizzoferrato and M. Casalboni, Appl. Phys. Lett. 73, 2378 (1998).

[11] R. Turton and M. Jaros, Appl. Phys. Lett. 69, 2891 (1996).

[12] P. A. M. Rodrigues, F. Cerdeira, J. C. Bean, Appl. Phys. Lett. 75, 145 (1999).

[13] The spectra shown on Figures 1 and 2 have not been corrected by the response of the detection system. On the other hand, the relative intensities listed in Table I were corrected for this experimental factor.

[14] See for example: J. Wagner, A. Dörnen, R. Sauer, Physical Review B 31, 5561 (1985) and J. Wagner, K. Thonke, R. Sauer, Physical Review B29, 7051 (1984).

[15] G. Davies, Physics Reports 176, 84 (1989). 